Artículo científico

Volumen 31(3):597-608. Septiembre-diciembre, 2020

e-ISSN 2215-3608, doi:10.15517/am.v31i3.40275

http://www.revistas.ucr.ac.cr/index.php/agromeso

\title{
Crecimiento de Trichoderma en rastrojo de piña para obtener esporas para uso agrícola ${ }^{1}$
}

\section{Trichoderma growth in pineapple stubble to obtain spores for agricultural use}

\author{
María Catalina Vega-Rodríguez ${ }^{2}$,Rodolfo Antonio Hernández-Chaverri ${ }^{3}$
}

1 Recepción: 9 de enero, 2020. Aceptación: 21 de mayo, 2020. Este trabajo formó parte del proyecto "Valorización de desechos agroindustriales para la producción de bioenergía y de enzimas de interés industrial aplicando técnicas biotecnológicas" bajo el financiamiento de la Universidad Estatal a Distancia (UNED), Costa Rica.

2 Universidad Estatal a Distancia, Escuela de Ciencias Exactas y Naturales. Programa de Investigación para la Promoción del Trabajo en Red. Unidad de Investigaciones Bioenergéticas y Ambientales. San José, Costa Rica. mariacatalina.vega@uned.cr (https://orcid.org/0000-00026730-8611).

3 Universidad Estatal a Distancia, Escuela de Ciencias Exactas y Naturales. Unidad de Investigaciones Bioenergéticas y Ambientales. Doctorado en Ciencias Naturales para el Desarrollo (DOCINADE-UNED-UNA-TEC). San José, Costa Rica. rohernandez@uned.ac.cr (https:// orcid.org/0000-0002-1841-1954).

\section{Resumen}

Introducción. El uso de controladores biológicos como alternativas a los productos químicos tradicionales resulta una opción ecológica y económica para los agricultores. Hongos del género Trichoderma son capaces de conferir resistencia a las plantas y aumentar la absorción de nutrientes, disminuyendo a su vez gran variedad de enfermedades en los cultivos. Objetivo. Evaluar los desechos de rastrojo de piña como sustrato para el crecimiento en fermentación sólida de dos cepas de Trichoderma para la producción de una solución de esporas con fines agrícolas. Materiales y métodos. El ensayo se llevó a cabo en el Laboratorio de Investigación en Química y Biociencias Aplicadas de la Universidad Técnica Nacional de Costa Rica, en el periodo entre abril del 2018 y octubre del 2019. Se utilizaron dos variantes del hongo T. viride y T. harzianum, estas se cultivaron en un medio natural de agar papa dextrosa (PDA) y rastrojo de piña. Se realizó un conteo de conidios en una cámara de Neubauer de 1/10 mm. Resultados. T. viride y T. harzianum presentaron un crecimiento uniforme en las placas de resiembra con cambios de coloración en el tiempo, de blanco a verde. T. viride presentó un mejor desarrollo en sustrato húmedo, mientras que T. harzianum en rastrojo con poca humedad. La concentración celular máxima en la solución líquida de T. harzianum y T. viride fue de 1132750 cél $\mathrm{ml}^{-1}$ y 1023250 cél ml$^{-1}$, respectivamente. Conclusión. Ambas cepas de Trichoderma se desarrollaron adecuadamente en el rastrojo de piña, sin embargo, el crecimiento eficiente de las cepas está asociado al manejo de factores externos como la cantidad de sustrato, oxígeno y humedad.

Palabras clave: conidios, desechos de piña, cinética de crecimiento, agroindustria.
Abstract
Introduction. The use of biological controllers as alternatives to traditional chemicals is an ecological and economic option for farmers. Fungi of the genus Trichoderma can confer resistance to plants and increase the 
absorption of nutrients, reducing a wide variety of crop diseases. Objective. To evaluate pineapple stubble wastes as a substrate for the solid fermentation growth of two Trichoderma strains to produce a spore solution for agricultural purposes. Materials and methods. The experiment was carried out at the Laboratory of Research in Chemistry and Applied Biosciences of the Universidad Tecnica Nacional of Costa Rica, in the period between April 2018 and October 2019. Two variants of fungus $T$. viride and T. harzianum were used, these were grown in natural potato dextrose agar (PDA) and pineapple stubble. A conidia count was performed in a 1/10 mm Neubauer chamber. Results. T. viride and T. harzianum showed uniform growth in reseeding plates with color changes in time, from white to green. T. viride showed better development in wet substrate, while T. harzianum in low moisture stubble. The maximum cell concentration in the liquid solution of T. harzianum and T. viride was $1132750 \mathrm{cell} \mathrm{ml}^{-1}$ and $1023250 \mathrm{cell} \mathrm{ml}^{-1}$, respectively. Conclusion. Both strains of Trichoderma developed adequately in the pineapple stubble, however, the efficient growth of the strains is associated with the management of external factors such as the amount of substrate, oxygen, and moisture.

Keywords: conidia, pineapple waste, growth kinetics, agroindustry.

\section{Introducción}

La demanda en la producción de cultivos en la industria alimentaria hace que diferentes organismos sean utilizados para el control biológico de fitopatógenos, como una alternativa para disminuir los costos de producción y reducir el impacto que causan los agroquímicos al ambiente (De-la-Cruz-Quiroz et al., 2019). El uso de agentes biológicos busca unificar los beneficios tanto protectores como de resistencia y desarrollo en los cultivos de interés, y a su vez incrementar el cultivo de especies simbiontes que por sí solas se encuentran en los suelos de manera natural (Moreno et al., 2015).

Algunos hongos del género Trichoderma (Hypocreaceae) tienen la capacidad de conferir a la planta cierta resistencia contra patógenos, ya que se asocian en distintas partes de la planta: raíz, flores, semillas y hojas (Martínez et al., 2013). Estos disminuyen los daños por patógenos en una amplia gama de cultivos; la asociación específica que se genera entre las hifas del hongo y la raíz de la planta se le conoce como micorriza (Kohler et al., 2015).

La capacidad de Trichoderma para generar micorrizas, les permite a las plantas ampliar su volumen radical para obtener más nutrientes (Sotomayor et al., 2019), lo cual confiere una mayor supervivencia en las plantaciones (Martínez et al., 2013; Sotomayor et al., 2019). Esta protección hace que la planta responda mejor a los cambios causados por factores externos en el suelo como la temperatura, salinidad y acidificación (Ghorbanpour et al., 2018) e incida en la productividad de esta (Martínez et al., 2019; Viera et al., 2019).

El empleo de Trichoderma en los cultivos favorece la elongación del tallo, el incremento del área foliar y la extensión radicular (Jacott et al., 2017), lo que permite mejorar el intercambio de nutrientes raíz-hongo-suelo, estas características le confieren al hongo la ventaja de desarrollarse bajo distintas condiciones ambientales, características que pueden considerarse para su cultivo masivo en condiciones in vitro.

El hongo Trichoderma presenta una facilidad para adaptarse a distintos sustratos, gran parte de sus requerimientos alimentarios se enfoca en propiedades de la biomasa como el almidón, pectina, aminoácidos y celulosa, en especial esta última (Arévalo et al., 2017). Sustratos como el arroz o la cascarilla de arroz, son empleados con frecuencia para el crecimiento de estos hongos, sin embargo, este tipo de biomasa contiene alrededor de $39 \%$ de celulosa (Dagnino et al., 2014), mientras que el rastrojo de piña puede tener hasta un $80 \%$ (Irías y Lutz, 2014), lo cual favorece el desarrollo de Trichoderma en fermentación sólida. Otras variables que se 
deben considerar en el cultivo de Trichoderma son las fuentes de oxígeno por periodos cortos y la exposición de los cultivos a la luz. Estos elementos inducen la conidiación en el momento en que las cepas entren nuevamente en contacto con el aire, así como la conidiación se induce por fotoperiodos y se ve inhibida en las células hifales que se desarrollan en condiciones anaerobias, por lo tanto, la relación luz-oxígeno es fundamental en las etapas de desarrollo de ambas cepas inicial fotoreactora y la conidiación bajo procesos oxidativos (Steyaert et al., 2010).

La amplia gama nutricional que requiere el Trichoderma para su desarrollo (almidón, pectina, celulosa, aminoácidos, entre otras), facilita su crecimiento en sustratos variados, por lo que el cultivo de este hongo es práctico y eficiente. Su desarrollo se basa, principalmente, en la fermentación sólida utilizando sustratos de arroz, trigo y residuos agroindustriales; sin embargo, se puede emplear la fermentación líquida como: melaza y levadura o la combinación de ambas técnicas (Arévalo et al., 2017).

Los sustratos como arroz y trigo son esenciales en la alimentación humana, por lo que el uso intensivo de estos es costoso en comparación con el aprovechamiento de residuos agroindustriales en la producción de Trichoderma. Lo anterior, abre la oportunidad de usar los residuos agroindustriales, uno de ellos es el rastrojo de piña (Ananas comosus) con gran abundancia en Costa Rica y en zonas tropicales.

El rastrojo de piña es un desecho agroindustrial con un alto volumen de generación luego del ciclo de cultivo de la fruta (González, 2012), se estima que es cerca de 300 toneladas por hectárea; otras investigaciones recientes indican que la generación de rastrojo es de 249₫70 toneladas por hectárea (Hernández-Chaverri y Prado-Barragán, 2018). El rastrojo se dispone por secado químico o en verde con implicaciones para el ambiente, además, es un vector para la proliferación de plagas como la mosca del establo, esto solo cuando no se realiza ningún proceso de compostaje. El secado químico usa un herbicida como medio de secado, posteriormente se quema con fuego en el campo y se hace incorporación mecánica al suelo; el tratamiento en verde se refiere a la incorporación mecánica directa con tractor al suelo, estos procesos tienen costos que varían entre los $\$ 1000$ hasta $\$ 2400$, respectivamente, según las condiciones climáticas y preparación del terreno para el siguiente ciclo de siembra (Hernández-Chaverri y Prado-Barragán, 2018).

La disponibilidad de este tipo de biomasa y la experiencia que reporta Vargas et al. (2019) para la producción de un sustrato líquido a partir de rastrojo fresco para el crecimiento micelial del hongo Shiitake, hace que el rastrojo de piña tenga un potencial como sustrato para la producción de esporas del hongo Trichoderma. El presente estudio tuvo como objetivo evaluar los desechos de rastrojo de piña como sustrato para el crecimiento en fermentación sólida de dos cepas de Trichoderma para la producción de una solución de esporas con fines agrícolas.

\section{Materiales y métodos}

\section{Descripción y localización del experimento}

Todos los experimentos se llevaron a cabo en el Laboratorio de Investigación en Química y Biociencias Aplicadas de la Universidad Técnica Nacional, Alajuela, Costa Rica, en el periodo comprendido entre abril del 2018 y octubre del 2019. Las condiciones del laboratorio se mantuvieron con una humedad relativa controlada del $50-55 \%$ y se encuentra $960 \mathrm{msnm}$.

\section{Muestra de rastrojo de piña}

La muestra de rastrojo de piña fue suministrada por la Unidad de Investigaciones Bioenergéticas y Ambientales (UIBEA) de la Universidad Estatal a Distancia (UNED). La muestra era de rastrojo de piña compuesta por varias submuestras recolectadas en los cantones de San Carlos y Los Chiles; la cual se entregó por la UIBEA con una 
humedad inferior al $10 \%$ y un tamaño de partícula inferior a los $8 \mathrm{~mm}$. La verificación de la humedad se llevó a cabo en cada etapa a $105 \pm 3{ }^{\circ} \mathrm{C}$, por 4 h en un horno de convección (Sluiter et al., 2008).

\section{Propagación del hongo}

Se utilizaron dos cepas de Trichoderma: T. viride y T. harzianum; estas fueron facilitadas por Sigma Aldrich. Se prepararon cuatro placas Petri con agar papa dextrosa (PDA) en medio natural (Ramos-García et al., 2019), en las cuales se realizó la propagación de las cepas por duplicado, mediante la técnica de estriado por agotamiento. El tiempo de incubación fue de $72 \mathrm{~h}$ a $25{ }^{\circ} \mathrm{C}$. Posteriormente, se hizo una resiembra o pasaje con $1 \mathrm{~cm}^{2}$ del micelio y se colocó en el centro de dos placas con PDA para obtener un crecimiento uniforme de los hongos; el pasaje se realizó por duplicado y se mantuvo las mismas condiciones de cultivo para la resiembra.

\section{Inoculación del hongo sobre el rastrojo}

En una primera etapa para la inoculación del sustrato, este se esterilizó a $120^{\circ} \mathrm{C}$ por $15 \mathrm{~min}$ y se colocó $50 \mathrm{~g}$ (para cubrir el $50 \%$ del volumen) de rastrojo de piña en cuatro matraces de $500 \mathrm{ml}$ (dos por cada tipo de hongo). Se inoculó cada matraz con $2 \mathrm{~cm}^{2}$ de micelio cultivado. Se ajustó la humedad del rastrojo al hidratar con agua la muestra hasta que se alcanzó un valor entre 40-50 \% de humedad (Gato, 2013). En esta etapa se hizo un seguimiento de la cinética de crecimiento de los hongos por cerca de tres semanas de incubación a $25^{\circ} \mathrm{C}$. La construcción de la curva de crecimiento se hizo con el promedio de los datos obtenidos para ambos matraces de $T$. viride y T. harzianum, respectivamente.

En una segunda etapa se realizó una nueva inoculación del sustrato estéril a dos porcentajes de humedad: entre 20-30 \% para T. harzianum y el otro con una hidratación de la muestra similar al proceso descrito con anterioridad (60-70\%). Este proceso se llevó a cabo a $25^{\circ} \mathrm{C}$, durante 30 días, dando seguimiento únicamente visual del crecimiento de los Trichodermas. Posteriormente, se unificó el sustrato de cada matraz, por cepa, y se realizó un traspaso del rastrojo de piña inoculado al sistema de bolsa plásticas, en las condiciones del laboratorio durante 60 días y $25^{\circ} \mathrm{C}$ (Figura 1). Además, del rastrojo traspasado, se agregó entre 150-200 g de rastrojo de piña fresco a las bolsas para su colonización. El sistema plástico estaba compuesto por dos capas: a) la interna: bolsa plástica transparente, semiabierta, en esta se encontraba el sustrato inoculado, y b) la externa: bolsa plástica negra con orificios de ventilación de $1 \mathrm{~cm}^{2}$ dispuestos al azar, cerrada en su parte superior por una liga elástica. Ambas capas formaron el sistema y este se acondicionó a un fotoperiodo de cuatro horas luz. Se mantuvo una relación de humedad aproximadamente constante, de tal manera que para T. viride el contenido de humedad fuera una relación de 1:2 m/v (60-70 \% de humedad), mientras que para T. harzianum una relación de 3:1 m/v (20-30\% de humedad). Para mantener la humedad, cada dos o tres días se realizó una aspersión de agua destilada (3-5 ml).

Terminado el periodo de inoculación en bolsa plástica se realizó un lavado al rastrojo con 11 de agua destilada estéril a través de un tamiz de 0,5 mm; el líquido recolectado se almacenó en dos botellas de plástico blanco de $500 \mathrm{ml}$, para cada tipo de hongo, a las condiciones del laboratorio, durante diecisiés meses para realizar un conteo de esporas viables.

\section{Conteo de conidios}

Se realizó un conteo preliminar por cada cepa, con una repetición, para estimar si la concentración celular se

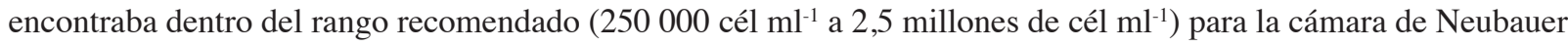
de 1/10 mm con profundidad de $10^{6}$ cél $\mathrm{ml}^{-1}$ (Bastidas, 2011). De tal manera, que el promedio de estos conteos 


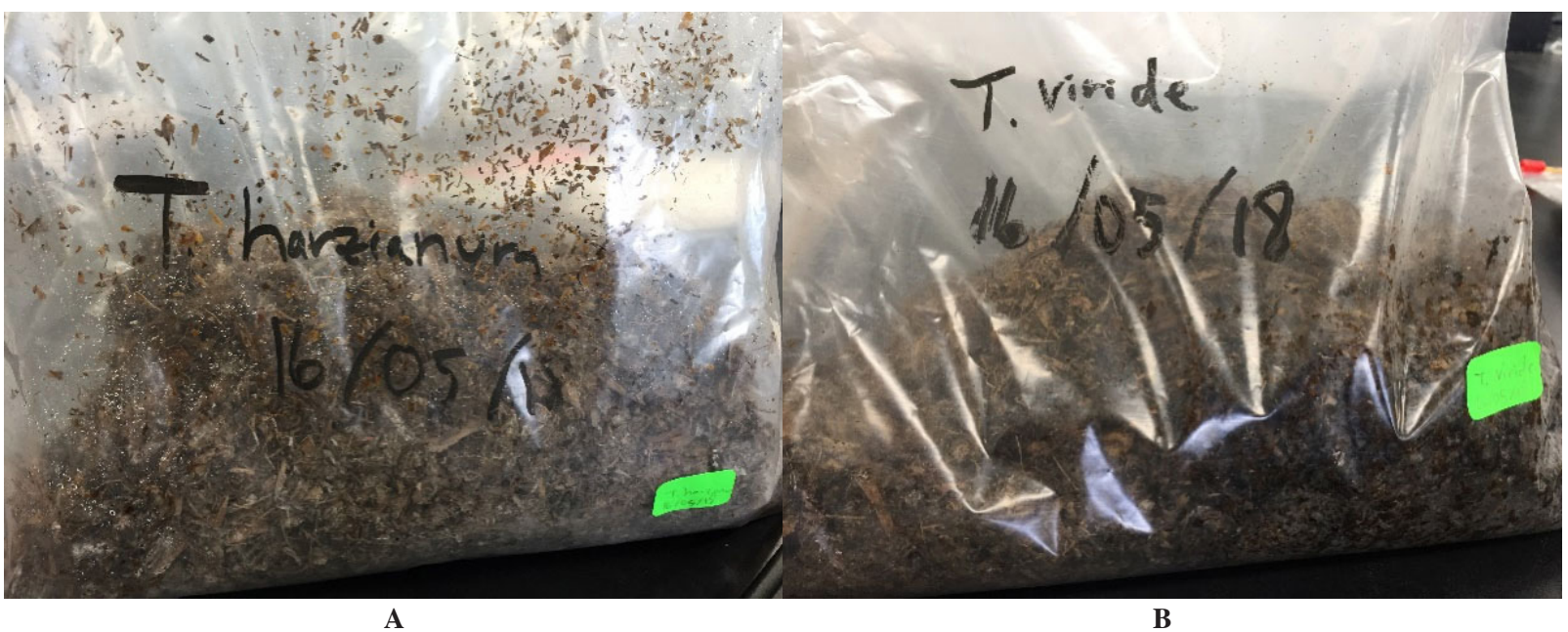

Figura 1. Sistema de bolsa plástica para el rastrojo de piña inoculado (A) T. harzianum, (B) T. viride. Laboratorio de Investigación en Química y Biociencias Aplicadas de la Universidad Técnica Nacional, Alajuela. Costa Rica. 2018. Fotografías por Hernández-Chaverri.

Figure 1. Plastic bag system for inoculated pineapple stubble (A) T. harzianum, (B) T. viride. Research Laboratory in Chemistry and Applied Biosciences of the National Technical University, Alajuela. Costa Rica. 2018. Photographs by Hernández-Chaverri.

preliminares para $T$. harzianum y T. viride fueron de 917500 cél ml $^{-1}$ y 1012500 cél ml-1 $^{-1}$, respectivamente. De acuerdo con las condiciones anteriores, no se utilizó un factor de dilución, debido a que la concentración de esporas estaba dentro de los rangos establecidos para el conteo. Se tomó una suspensión inicial de $3 \mathrm{ml}$ de cada una de las muestras almacenadas y de esta se tomó $20 \mu 1$ con una micropipeta con capacidad máxima $200 \mu 1$ y se colocó en la cámara por medio de capilaridad. Para la observación de la muestra se utilizó un microscopio óptico bajo un aumento de 40 X marca OPTIMA G-32.

El conteo se determinó mediante la observación de células vivas y muertas, tomando en cuenta la diferenciación de color natural, donde el color café oscuro representó las células muertas e hialino las vivas. Esta observación, se realizó únicamente en la celda central de veinticinco cuadros de $0,2 \mathrm{~mm}$, con cinco cuadros de manera diagonal de izquierda a derecha (Figura 2); dentro del recuento se tomó en consideración las células observadas dentro de los bordes límite de cada cuadro $(4 \times 4)$ que estuvieran en el borde superior y el borde izquierdo sin sobrepasar las líneas triples del cuadro (UASLP, 2013).

Se determinó la concentración celular de la suspensión inicial con base en la fórmula de UASLP (2013):

Concentración celular $=$ células vivas $\div 100 \times$ factor de dilución $\times 10000$.

Se realizó un total de veintidós conteos (más el ensayo preliminar) a lo largo del experimento con una frecuencia de 15-20 días para cada recipiente plástico con la suspensión y por duplicado. Se trabajó con el promedio general para cada especie. Para obtener la cantidad de células totales en el cultivo inicial de rastrojo de piña, se multiplicó la concentración celular por el volumen total de la muestra inicial, mientras que el porcentaje de viabilidad celular se determinó a partir de la división del total de células vivas entre el total de células (vivas y muertas). Los conteos se realizaron por duplicado en cada frasco y se promediaron las lecturas de cada frasco según la especie de Trichoderma. 


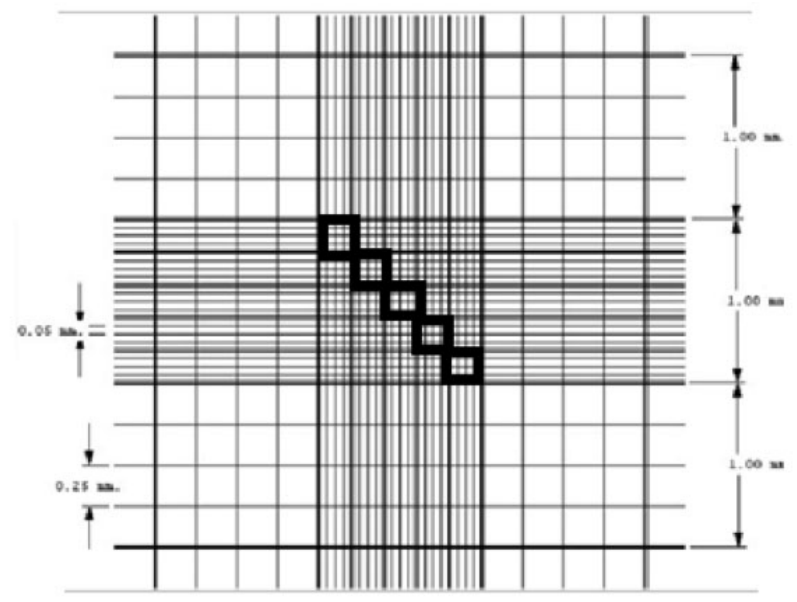

A

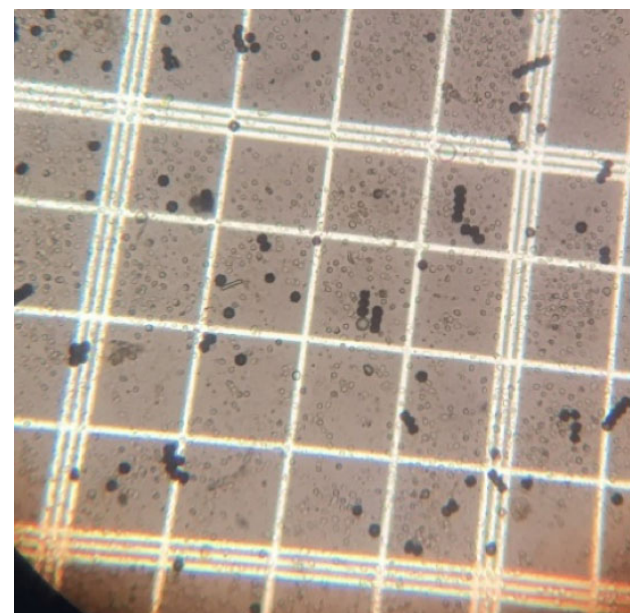

B

Figura 2. (A) Método de conteo de conidios en la rejilla de la cámara de Neubauer 1/10mm UASLP (2013), (B) fotografía de una rejilla de conteo con conidios muertos de $T$. viride (coloración oscura) y vivos. Laboratorio de Investigación en Química y Biociencias Aplicadas de la Universidad Técnica Nacional, Alajuela. Costa Rica. 2018. Fotografía por Vega-Rodríguez.

Figure 2 (A) Method of counting conidia in the grid of the Neubauer chamber 1/10mm UASLP (2013), (B) photograph of a counting grid with dead of $T$. viride (dark coloration) and live conidia. Research Laboratory in Chemistry and Applied Biosciences of the National Technical University, Alajuela. Costa Rica. 2018. Photograph by Vega-Rodríguez.

\section{Análisis estadístico}

Se realizó un ANOVA de una vía para establecer diferencias de medias en: la concentración celular $\left(\right.$ cell $\left.^{\mathrm{ml}^{-1}}\right)$, las células totales (cell) y el porcentaje de viabilidad, con un nivel de significancia del $95 \%$.

\section{Resultados}

\section{Incremento del inóculo}

Para el hongo T. viride se evidenció una morfología macroscópica con colonias filamentosas al inicio de la siembra y más compactas pasados tres días de cultivo; fecha en la cual se empezó a observar una esporulación de color verde con textura granular y crecimiento concéntrico. A nivel microscópico, se observaron hifas hialinas septadas, conidióforos hialinos ramificados y conidios de forma redonda u ovalada de paredes rugosas. En $T$. harzianum, al igual que en $T$. viride, se presentó un cambio de coloración en las colonias de blanco a verde a partir del día 3 desde la siembra. Se observó a nivel microscópico (T. harzianum), hifas hialinas, septadas y ramificadas con conidióforos ramificados de forma piramidal y conidios ovoides de paredes lisas. Fue posible verificar esta descripción con la reportada para este tipo de cepas en Vásquez-Cárdenas (2010) y Kubicek y Harman (1998).

\section{Cinética de crecimiento}

Una tendencia creciente para ambos tipos de Trichoderma en su propagación sobre el rastrojo de piña se muestra en la Figura 3. Esta tendencia se describe lineal como se muestra en el Cuadro 1, con un $R^{2}$ mayor a 0,92 para los dos tipos de hongo. 


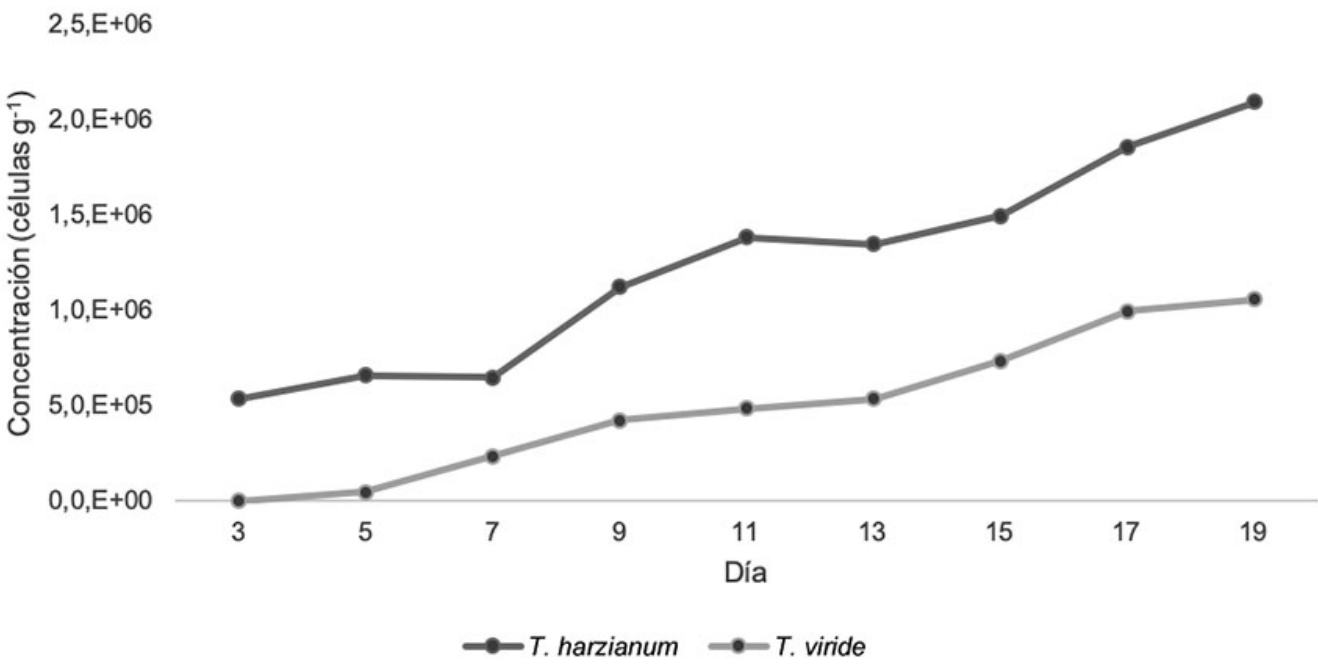

Figura 3. Crecimiento para las dos cepas de Trichoderma: T. viride y T. harzianum, con 19 días de incubación. Laboratorio de Investigación en Química y Biociencias Aplicadas de la Universidad Técnica Nacional, Alajuela. Costa Rica. 2018.

Figure 3. Growth for the two Trichoderma strains: T. viride and T. harzianum, with 19 days of incubation. Research Laboratory in Chemistry and Applied Biosciences of the National Technical University, Alajuela. Costa Rica. 2018.

Cuadro 1. Modelo de ajuste lineal para el crecimiento de las dos cepas de Trichoderma: T. viride y T. harzianum, sobre rastrojo de piña. Laboratorio de Investigación en Química y Biociencias Aplicadas de la Universidad Técnica Nacional, Alajuela. Costa Rica. 2018.

Table 1. Linear adjustment model for the growth of the two Trichoderma strains: T. viride and T. harzianum, on pineapple stubble. Research Laboratory in Chemistry and Applied Biosciences of the National Technical University, Alajuela. Costa Rica. 2018.

\begin{tabular}{ccc}
\hline Cepas & Modelo de ajuste lineal & $\mathbf{R}^{\mathbf{2}}$ \\
\hline T. viride & $196250 \cdot \mathrm{X}+260417$ & 0,956 \\
T. harzianum & $136875 \cdot \mathrm{X}+180208$ & 0,976 \\
\hline
\end{tabular}

\section{Inoculación del hongo sobre el rastrojo}

T. viride tuvo un crecimiento más acelerado en el sustrato húmedo para una relación 1:2 m/v, mientras que $T$. harzianum se desarrolló mejor en el rastrojo de piña con poca humedad, en relación 3:1 m/v. La cantidad de sustrato en los sistemas aireados fue un factor relevante en la producción de esporas de ambas cepas, ya que se observó una menor esporulación cuando las bolsas se encontraban a >50\% de capacidad.

\section{Conteo de conidios}

Posterior al día 257 y hasta el día 480, el porcentaje de viabilidad fue relativamente constante durante ese periodo (223 días), a pesar de que se mantuvo las mismas condiciones mecánicas en todos los conteos (Figura 4).

La concentración celular de $T$. harzianum presentó su valor más bajo para el día 84 con una concentración de 322000 cél ml $^{-1}$, mientras que la mayor concentración se produjo para el día 480 con un valor de 1132750 cél ml $^{-1}$. 


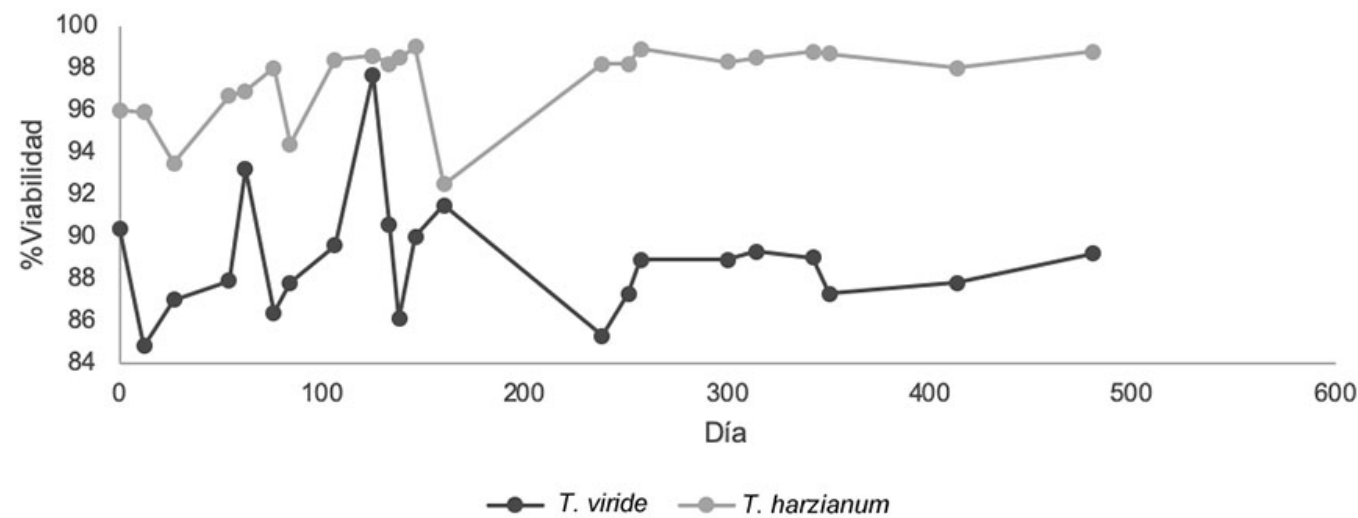

Figura 4. Viabilidad porcentual de células de las dos cepas de Trichoderma: T. viride y T. harzianum durante el periodo comprendido entre abril del 2018 y octubre del 2019. Laboratorio de Investigación en Química y Biociencias Aplicadas de la Universidad Técnica Nacional, Alajuela. Costa Rica. 2018-2019.

Figure 4. Percentage viability of cells of the two Trichoderma strains: T. viride and T. harzianum during the period between April 2018 and October 2019. Research Laboratory in Chemistry and Applied Biosciences of the National Technical University, Alajuela. Costa Rica. 2018-2019.

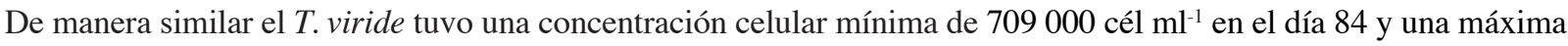
de 1023250 cél ml$^{-1}$ en el día 106 (Figura 5).

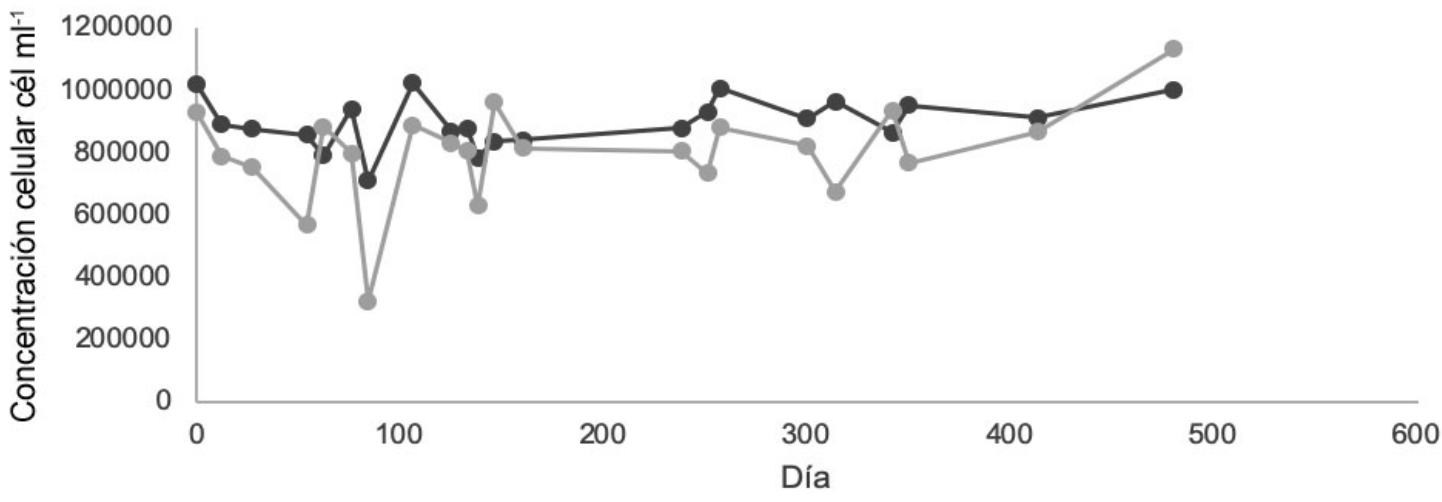

$\longrightarrow T$. viride $\longrightarrow T$. harzianum

Figura 5. Concentración celular de las dos cepas de Trichoderma: T. viride y T. harzianum durante el periodo comprendido entre abril del 2018 y octubre del 2019. Laboratorio de Investigación en Química y Biociencias Aplicadas de la Universidad Técnica Nacional, Alajuela. Costa Rica. 2018-2019.

Figure 5. Cell concentration of the two Trichoderma strains: T. viride and T. harzianum during the period between April 2018 and October 2019. Research Laboratory in Chemistry and Applied Biosciences of the National Technical University, Alajuela. Costa Rica. 2018-2019.

Se logró determinar que existe diferencia significativa en el porcentaje de viabilidad de T. viride y $T$. harzianum $(\mathrm{p}<0,05)$, así como, para los parámetros de concentración y células totales $(\mathrm{p}<0,05)$, durante el periodo de estudio (Cuadro 2). 
Vega-Rodríguez y Hernández-Chaverri: Solución de Trichoderma de uso agrícola

Cuadro 2. Datos comparativos para las dos cepas de Trichoderma: T. viride y T. harzianum, durante un periodo de 480 días. Laboratorio de Investigación en Química y Biociencias Aplicadas de la Universidad Técnica Nacional, Alajuela. Costa Rica. $2018-2019$.

Table 2. Comparative data for the two Trichoderma strains: T. viride and T. harzianum, over a period of 480 days. Research Laboratory in Chemistry and Applied Biosciences of the National Technical University, Alajuela. Costa Rica. 2018-2019.

\begin{tabular}{lccc}
\hline & \multicolumn{3}{c}{ Cepas } \\
\cline { 2 - 4 } & T. viride & T. harzianum & p-value \\
\hline Concentración (cél. ml ${ }^{-1}$ ) & $896568 \pm 80563$ & $799114 \pm 159464$ & 0,014 \\
Células totales (cél.) & $2689705 \pm 241688$ & $2397341 \pm 478391$ & 0,014 \\
\% viabilidad & $88,9 \pm 2,8$ & $97,4 \pm 1,8$ & 0,000 \\
\hline
\end{tabular}

\section{Discusión}

En términos generales, las dos cepas de Trichoderma (T. viride y T. harzianum) presentaron un crecimiento positivo en el rastrojo de piña tanto en los ensayos con matraces como en los sistemas plásticos aireados. La cinética de crecimiento llevó un comportamiento lineal constante en ambos Trichodermas, pero hubo diferencia en la velocidad del $T$. viride con respecto al $T$. harzianum, la cual se asocia con la adaptabilidad del hongo con el sustrato (Allori et al., 2017). Otro elemento para considerar fue la cantidad de rastrojo de piña empleado y su distribución en el medio (matraz o bolsa), que puede ser un factor que determinó la aceleración del crecimiento de las cepas (Steyaert et al., 2010). Lo anterior, se debe a que el oxígeno es una variable importante en el crecimiento conidial y al ir creciendo la biomasa del hongo se ocluyen los espacios para el flujo de oxígeno u dióxido de carbono, por lo que generar movimientos esporádicos del sistema (sustrato) favorece la circulación del aire en la bolsa o matraz y evita la acumulación de dióxido de carbono (Allori et al., 2017); factor inhibitorio del crecimiento de varias especies de Trichoderma y determinante para la conidiación de estos hongos (Singh et al., 2014). Este fenómeno se logró evidenciar en el conteo número 5, en el cual el porcentaje de viabilidad aumentó en ambas cepas tras haber mantenido cerrados los sistemas de almacenamiento durante un periodo de veintisiete días.

La ausencia o presencia de oxígeno estuvo reflejada en el porcentaje de viabilidad de las cepas, ya que los recipientes plásticos se llenaron a una capacidad del $90 \%$ con la solución de esporas y se mantuvo un $10 \%$ para la contención de aire, de tal manera que al momento de abrir el frasco contenedor para realizar el conteo de conidios, por un periodo aproximado de dos minutos por extracción, se equilibró el contenido de oxígeno, el cual permitió mantener activa la conidiación y por ende, se generó un equilibrio en la concentración celular lo cual favoreció la viabilidad de las esporas. Cuando se determina el porcentaje de viabilidad con pocos días de diferencia entre cada conteo, fue posible observar un aumento tanto en la viabilidad como en la concentración celular (Figura 4 y 5), debido al ingreso de oxígeno durante esos periodos.

Se logró mantener la concentración y la viabilidad de las esporas durante un periodo prolongado (480 días) a pesar de que el sistema de almacenamiento no contenía nutrientes adicionados, únicamente agua destilada. Lo anterior, se debió a que estos hongos poseen dentro de su estructura una pared celular gruesa que les permite mantenerse aislados del ambiente hasta que las condiciones del medio sean apropiadas para su desarrollo, condición conocida como dormancia (Allori et al., 2017).

La concentración celular y la viabilidad de las cepas en el tiempo son parámetros que se utilizan para determinar la calidad de los productos formulados. Ambas especies de Trichoderma presentaron resultados estables en la viabilidad durante el periodo, no obstante, Viera et al. (2018) indicaron que las formulaciones a partir de aceites se comportan mejor en la agricultura, ya que presenta una vida útil prolongada en las especies vegetales. Sin embargo, no descarta las formulaciones líquidas para la producción de Trichoderma, debido a que puede haber variaciones en el comportamiento de las cepas del mismo género; en este caso los investigadores evaluaron los parámetros con 
T. asperellum y Purpureocillium lilacinum. Esta diferencia en los parámetros se observó en T. viride, en la cual el porcentaje de viabilidad disminuyó $1,2 \%$ respecto a la muestra inicial, mientras que $T$. harzianum aumento $2,8 \%$ en comparación con el día 0 .

T. viride disminuyó su concentración en 0,08 \% y T. harzianum tuvo un aumento de 1,16\% en la concentración celular, ambos valores representan la diferencia de la última muestra respecto a la muestra inicial. La concentración mínima a la cual debe estar el Trichoderma es de $2 \times 10^{6} \mathrm{UFC} \mathrm{g}^{-1}$ en un medio selectivo (Viera et al., 2018). La solución de contención de ambas cepas era agua destilada con el objetivo de evaluar su comportamiento en el tiempo, no obstante, para lograr una concentración con un aumento significativo, es fundamental contar con un medio selectivo y añadir nutrientes en las formulaciones. Además, utilizar agua destilada estéril como medio para la conservación de esporas, resulta efectivo y económico, ya que suprime los cambios morfológicos del hongo (Gato, 2013), asegura la resistencia durante periodos extensos y previene la generación de pleomorfismos.

Realizar un proceso de fermentación sólida previo al almacenamiento líquido de las cepas, es favorable para obtener gran cantidad de biomasa fúngica. Estas cepas presentan alta tolerancia en condiciones limitadas de agua, incentiva la secreción de enzimas hidrolíticas y acelera el proceso de conidiogénesis, elementos asociados al tipo de sustrato y al tamaño de partícula (Arévalo et al., 2017). Si bien solo hay diferencia significativa en la viabilidad por tipo de Trichoderna, no se encontró evidencia en la diferencia de concentración y cantidad de células totales en las lecturas de la solución líquida. Esto apunta que en nuevas investigaciones es posible mejorar el medio de la solución y la frecuencia de intercambio de gases $\left(\mathrm{O}_{2}, \mathrm{CO}_{2}\right)$.

\section{Conclusiones}

Las dos cepas de Trichoderma (T. viride y T. harzianum) se desarrollaron adecuadamente en el rastrojo de piña, sin embargo, T. viride necesitó condiciones de mayor humedad en el medio que T. harzanium para su crecimiento.

La concentración celular en las dos cepas de Trichoderma (T. viride y T. harzianum) se mantuvo relativamente estable durante un periodo extenso sin necesidad de añadir nutrientes al medio. T. viride presentó un promedio de concentración celular mayor que T. harzianum. El porcentaje de viabilidad promedio se mantuvo por encima del $88 \%$, en ambas cepas; sin embargo, T. harzianum demostró una viabilidad más estable, alrededor del $97 \%$, a lo largo del tiempo en comparación con $T$. viride.

El rastrojo de piña fue un buen sustrato para el crecimiento de Trichoderma, debido a su alta disponibilidad durante el año, es un recurso de bajo costo al ser un desecho agroindustrial y mostró ser un buen medio para la fermentación sólida para los hongos en estudio. Sin embargo, se debe profundizar más en la investigación del rastrojo como sustrato al considerar otras variables como tamaño de partícula, empaquetado del sistema y transferencias de gases.

\section{Agradecimientos}

Los autores expresan su agradecimiento al Programa de Investigación para la Promoción del Trabajo en Red por su apoyo para ser parte del grupo de estudiantes vinculados a investigación de la UNED. Al Dr. Maynor Vargas Vargas del Laboratorio de Biociencias y Química Aplicada de la Universidad Técnica Nacional por todas las facilidades prestadas para llevar a cabo esta investigación. Proyecto de Mejoramiento Institucional (AMI-UNED) por el apoyo brindado. 


\section{Literatura citada}

Allori, E., M. Yasem de Romero, y L. Ploper. 2017. Evaluación de sustratos para la producción de esporas de Trichoderma y estudio del crecimiento en arroz de las cepas antagonistas TPT03, TPT02, MRT35 y MRT40. Rev. Agron. Noroeste Arg. 37(1):57-66.

Arévalo, E., J. Cayotopa, D. Olivera, M. Gárate, E. Trigoso, D.B. Costa, y B. Leon. 2017. Optimización de sustratos para la producción de conidias de Trichoderma harzianum. Por fermentación sólida en la región de San Martín. Perú. Rev. Investig. Altoandin. 19:135-144. doi:10.18271/ria.2017.272

Bastidas, O. 2011. Conteo celular con hematocitómetro: Uso elemental del hematocitómetro. SCRIBD, San Francisco, CA, USA. https://www.scribd.com/document/105937668/Conteo-Camara-Neubauer (consultado 3 ene. 2020).

Dagnino, E.P., Chamorro, E.R., Felissia, F.E., y M.C. Area. 2014. Obtención de bietanol a partir de la celulosa presente en cascarilla de arroz y aserrín de algarrobo. Avanc. Energ. Renov. Med. Amb. 18(1):1-7.

De-la-Cruz-Quiroz, R., S. Roussos, M.T. Tranier, R. Rodríguez-Herrera, N. Ramírez-Guzmán, and C.N. Aguilar. 2019. Fungal Spores Production by solid-state fermentation under hydric stress condition. Rev. Cient. Univ. Auton. Coahuila 11(21):2-6.

Gato, Y. 2013. Métodos de conservación y formulación de Trichoderma harzianum Rifai. Fitosanidad 14:189-195.

Ghorbanpour, A., A. Salimi, M. Ghanbary, H. Pirdashti, and A. Dehestani. 2018. The effect of Trichoderma harzianum in mitigating low temperature stress in tomato (Solanum lycopersicum L.) plants. Sci. Hort. 230:134-141. doi:10.1016/j. scienta.2017.11.028

González, L.A. 2012. Manual técnico para el manejo de rastrojos en el cultivo de piña. MAG, CRI. http://www.mag.go.cr/ bibliotecavirtual/Q70-10493.pdf (consultado 3 ene. 2020).

Hernández-Chaverri, R.A., y L.A. Prado-Barragán. 2018. Impacto y oportunidades de biorrefinería de los desechos agrícolas del cultivo de piña (Ananas comosus) en Costa Rica. Cuadernos Invest. 10:455-468. doi:10.22458/urj.v10i2.2059

Irías, A., y G. Lutz. 2014. Composición química de la biomasa residual de la planta de piña variedad MD2 proveniente de Guácimo, Limón. Cienc. Tecnol. 30(2):27-34.

Jacott, C.N., J.D. Murray, and C.J. Ridout. 2017. Trade-offs in arbuscular mycorrhizal symbiosis: disease resistance, growth responses and perspectives for crop breeding. Agronomy 7(4):75. doi:10.3390/agronomy7040075

Kohler, A., A. Kuo, L. Nagy, E. Morin, K. Barry, F. Buscot, and J. Colpaert. 2015. Convergent losses of decay mechanisms and rapid turnover of symbiosis genes in mycorrhizal mutualists. Nat. Gen. 47:410-415. doi:10.1038/ng.3223

Kubicek, C.P., and G.E. Harman.1998. Trichoderma and Gliocladium. Volume 1: Basic biology, taxonomy and genetics. Google, USA. https://books.google.co.cr/books?id=9ymnPzUM-w0C\&printsec=frontcover\&hl=es\#v=onepage \&q\&f=fa lse (accessed May 14, 2020).

Martínez, B., D. Infante, y Y. Reyes. 2013. Trichoderma spp. y su función en el control de plagas en los cultivos. Rev. Protec. Veg. 28(1):1-11.

Martínez, A., L. Villacís, E. Viera, R. Jácome, M. Espín, O. León, y R. Santana. 2019. Evaluación de nuevas tecnologías de producción limpia de la mora de castilla (Rubus glaucus Benth), en la zona Andina de Ecuador, para un buen vivir de los fruticultores. J. Selva Andina Biosph. 7(1):63-70.

Moreno, M.V., C. Merlos, L. Silvestro, H. Forján, y M.L. Manso. 2015. Hongos de suelo en sistemas agrícolas. AgroBarrow $56: 20-22$ 
Ramos-García, M., Aparicio-García, P. F., Bautista-Baños, S., and M. Hernández-López. 2019. Efecto de diferentes medios de cultivo sobre la patogenicidad de Aspergillus flavus en frutos de higo. Rev. Mex. Fitopatol. 37(1):8-13.

Singh, A., M. Shahid, M. Srivastava, S. Pandey, A. Sharma, and V. Kumar. 2014. Optimal physical parameters for growth of Trichoderma species at varying pH, temperature and agitation. Virol. Mycol. 3:127. doi:10.4172/2161-0517.1000127

Sluiter, A., B. Hames, D. Hyman, C. Payne, R. Ruiz, C. Scarlata, J. Sluiter, D. Templeton, and J. Wolfe. 2008. Determination of total solids in biomass and total dissolved solids in liquid process samples. Laboratory Analytical Procedure (LAP). National Renewable Energy Laboratory, USA. https://www.nrel.gov/docs/gen/fy08/42621.pdf (accessed May 14, 2020).

Sotomayor, A., A. González, J. Kang, A. Villavicencio, T. Jackson, and W. Viera. 2019. Effect of the application of microorganisms on the nutrient absorption in avocado (Persea americana Mill.) seedlings. J. Korean Soc. Inter. Agric. 31(1):17-24.

Steyaert, J.M., R.J. Weld, A. Mendoza-Mendoza, and A. Stewart. 2010. Reproduction without sex: conidiation in the filamentous fungus Trichoderma. Microbiology 156:2887-2900. doi:10.1099/mic.0.041715-0

UASLP (Universidad Autónoma de San Luis Potosí). 2013. Conteo celular y evaluación de viabilidad. SCRIBD, San Francisco, CA, USA. https://www.scribd.com/document/348653988/Cell-Counts (consultado 9 ene. 2020).

Vargas, M., R. Hernández, y A. Jiménez. 2019. Caracterización de la biomasa de piña (Ananas comosus) y su valoración en la propagación micelial del hongo shiitake (Lentinula edodes). Yulök Rev. Inov. Acad. 3(1):13-27.

Vásquez-Cárdenas, J.A. 2010. Caracterización microbiológica y producción de Trichoderma harzianum y Trichoderma viride en cultivo artesanal. Universidad Javeriana, Bogotá, COL.

Viera, W., M. Noboa, J., Bermeo, F. Báez, y T. Jackson. 2018. Parámetros de calidad de cuatro tipos de formulaciones a base de Trichoderma asperellum y Purpuricillium lilacinum. Enfoque UTE 9(4):145-153.

Viera, W., M. Noboa, A. Martínez, F. Báez, R. Jácome, L. Medina, and T. Jackson. 2019. Trichoderma asperellum increases crop yield and fruit weight of blackberry (Rubus glaucus) under subtropical Andean conditions. Vegetos 32:209-215. 